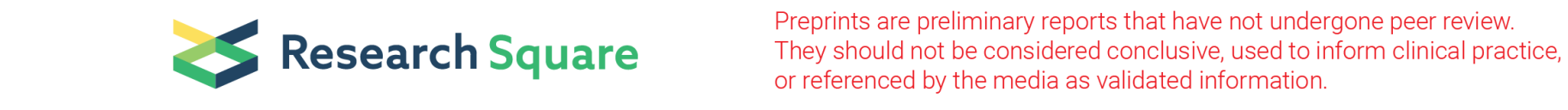

\title{
Identification of appropriate reference genes for gene expression studies in mice left ventricles from different developmental stages
}

Jie Ren ( $\sim$ renjie_2016@126.com )

Chinese Academy of Medical Sciences \&amp; Peking Union Medical College Fuwai Hospital https://orcid.org/0000-0002-45953281

Ningning Zhang

Fuwai Hospital State Key Laboratory of Cardiovascular Disease

\section{Xiangjie Li}

Fuwai Hospital State Key Laboratory of Cardiovascular Disease

\section{Xiaogang Sun}

National Center for Cardiovascular Diseases China: Chinese Academy of Medical Sciences \&amp; Peking Union Medical College Fuwai Hospital

\section{Jiangping Song}

Fuwai Hospital State Key Laboratory of Cardiovascular Disease

\section{Research Article}

Keywords: Reference genes, Heart, Development, Real-time quantitative polymerase chain reaction (RT-qPCR), Stability

Posted Date: February 19th, 2021

DOI: https://doi.org/10.21203/rs.3.rs-208040/v1

License: (c) (i) This work is licensed under a Creative Commons Attribution 4.0 International License. Read Full License 


\section{Abstract}

Aims: Real-time quantitative polymerase chain reaction (RT-qPCR) is the standard assay used for revealing the gene expression characteristics. However, the RT-qPCR studies all need reference genes for normalization to make the results comparable, which should hold a high expression stability during all experimental datasets. So far, there was no optimal set of reference genes identified in mice left ventricles (LV) across embryonic and postnatal stages. The objective of our research was to identify the appropriate reference genes in mice LV from different developmental stages.

Methods and Results: we investigated the gene expressions of common 21 candidate housekeeping genes in mice LV from 7 different developmental stages, almost throughout the whole period of the mouse lifespan. The expression of some candidate reference genes, such as $18 S$ and $A c t b$, apparently fluctuated. The stability of potential reference genes was evaluated by a number of methods, such as GeNorm, NormFinder, BestKeeper, Delta-Ct and RefFinder method. we identified a set of optimal reference genes that can be reliably used for normalization of RT-qPCR experiments in different developmental stages of mice LV. Our results showed that following genes should not be used as reference genes in mice LV development studies: 18S, Hmbs, Ubc, Psmb4, Tfrc and Actb. And the RplpO appeared to represent a good choice.

Conclusions: Our study provides the expression stability of the commonly used reference genes in process of LV development and maturation. We also identified a set of optimal reference genes under different conditions. Our findings may be helpful in future studies to investigate the gene expression patterns and mechanism of mammalian heart development.

\section{Introduction}

The heart is the first organ to develop in the embryo, which held significantly different physiological function and gene expression profiling among different developmental stages[1]. Cardiac malformation is the most common form of congenital anomalies associated with a serious societal impact because of the significant morbidity and mortality[2]. Meanwhile, multiple studies have found that the distinct gene expression pattern during cardiac maturation[3,4]. The main function of the heart, pumping blood to make a constant supply of oxygen and nutrients for body, is mainly determined by the left ventricular (LV) status[5,6]. And the LV remodeling is the major manifestations of many cardiac disorders[7,8]. However, the specific molecular mechanism involved in LV development/maturation remained not fully understanding[9].

Mice have been used frequently as a model in the area of cardiac development/maturation, because of the similar anatomical, genetical and physiological characteristics[10]. Transcriptome analysis have been confirmed to providing a broad insight into the molecular regulatory networks that underlie stage-specific differences in mice hearts[11,12]. Until today real-time quantitative polymerase chain reaction (RT-qPCR) is the standard assay used for gene expression quantification analysis with high sensitivity and accuracy[13]. However, the RT-qPCR studies all need reference genes for normalization to make the results comparable among different groups[14]. The unstable reference genes would lead to erroneous results, which makes the selection of the internal reference genes an important factor in the design of a RT-qPCR experiment[15]. The reference genes should hold a high expression stability during all experimental datasets[16,17]. So far, there was no ideal reference gene that is appropriate for all kinds of conditions[18]. Thus, it is critical to identify the reference genes that have relatively stable expression in the specific context of study.

The common reference genes used in RT-qPCR for heart samples were Glyceraldehyde-3-phosphate dehydrogenase (Gapdh), 18S ribosomal RNA (18S), and actin beta (Actb)[19]. And the other genes also have been used as reference genes in several studies[17]. But not all are suitable for all experimental conditions or different developmental samples[20]. Some housekeeping genes were demonstrated significantly altered in heart development or maturation process[21]. A recent study identified the appropriate reference genes for normalizing gene expression data of heart with different developmental stages in mouse[22]. So far, there was little optimal set of reference genes had been identified in LV tissues across embryonic and postnatal stages in mice.

Our work systematically assessed the expression stability of the commonly used reference genes $(n=21)$ in mice LV acorss heart development and maturation. Synthesizing the results of a number of methods, such as GeNorm[21], NormFinder[23], BestKeeper[24], Delta-Ct[25] and RefFinder[26] method, we identified a set of optimal reference genes that can be reliably used for normalization of RT-qPCR experiments in different specific conditions.

Page $2 / 20$ 


\section{Methods}

\section{Sample collection}

The C57BL/ 6 mice were maintained in plastic cages at 23 to $25^{\circ} \mathrm{C}$ with a 12/12-hour light/dark cycle. The protocol for the experiment was approved by the Animal Care and Use Committee of Fuwai hospital. The animals were mated overnight and pregnancy was detected at 0.5 gestation day by the observation of the mating plug. The mice of various ages were euthanized by CO2 inhalation to collect LV specimens (Figure 1). The mouse embryonic hearts were collected when the pregnant female mice were sacrificed on exact gestation day. LV in embryonic hearts were identified and collected by careful microdissection under stereomicroscope (Leica) using sharpened microdissection scissors. Tissue from 10 to 15 embryos were pooled in one tube to ensure a sufficient final RNA per sample, so did in mice within 7 days of birth.

\section{RNA isolation and CDNA synthesis}

Each RNA isolation was performed from $30 \mathrm{mg}$ of the frozen LV tissue. MagNA Lyser Green Beads (Roche, Switzerland) containing TRIzol (Invitrogen, USA) and the MagNA lyser instrument (Roche, Switzerland) were used for tissue homogenate. Then the standard instructions of Trizol manufacturer were carried out for total RNA extraction. The concentration and quality were evaluated by NanoDrop2000 (NanoDrop Technology, USA). For each sample, First Strand cDNA was synthesized from 500ng total RNA by using the Takara Reverse Transcription Kit (Takara, Japan). All samples within this experiment were processed simultaneously to avoid interexperimental variations.

\section{Selection of candidate reference genes and Primer design}

In this study, the 21 housekeeping genes (Table 1), which were commonly used as reference genes in previous cardiac studies, were analyzed to provide a better reference guide for identification of molecular mechanisms underlying cardiac development and maturation. Those candidate genes included the Actb (Actin beta), Gapdh (Glyceraldehyde-3-phosphate dehydrogenase), Reep5 (Receptor accessory protein5), (Ribosomal protein L5), Psmb4 (Proteasome subunit beta 4), Vcp (Valosin containing protein), B2m (Beta-2-microglobulin), Gusb (Glucuronidase beta), Hmbs (Hydroxymethylbilane synthase), Hprt1 (Hypoxanthine phosphoribosyltransferase 1), Ipo8 (Importin 8), Pgk1 (Phosphoglycerate kinase 1), Polr2a (RNA polymerase II subunit A), Ppia (Peptidylprolyl isomerase A), RplpO (Ribosomal protein lateral stalk subunit P0), Tbp (TATA box binding protein), Tfrc (Transferrin receptor), Ubc (Ubiquitin C), Ywhaz (14-3-3 protein zeta), 18S (eukaryotic 18S ribosomal RNA) and Sdha (succinate dehydrogenase complex flavoprotein subunit A). According to the published mouse sequences, the NCBI primer designing tool was used to generate the RT-qPCR primers sequences (Table 1).

\section{RT-qPCR}

RT-qPCR was performed in 384 well plates using the Viia7 Real Time PCR System (Applied Biosystems). Each $10 \mu$ reaction mixture contained the $5 \mu \mathrm{l} 2 \times$ SYBR Green Real-Time PCR Master Mix reaction mixture, $0.4 \mu \mathrm{L}$ of each primer $(10 \mu \mathrm{M}), 2 \mu \mathrm{l}$ cDNA $(2.5 \mathrm{ng} / \mu \mathrm{l})$ and $2.2 \mu \mathrm{L}$ double-distilled water. The thermal cycling program: $95^{\circ} \mathrm{C}$ for $10 \mathrm{~min}$, followed by 40 cycles of $15 \mathrm{~s}$ at $95^{\circ} \mathrm{C}$ and $1 \mathrm{~min}$ at $60^{\circ} \mathrm{C}$. Each sample was performed in three technical replicates and the resulting Ct values averaged.

\section{Evaluation of Expression Stability}

The gene expression stability was evaluated by analyzing the raw $\mathrm{Ct}$ values in the four independent statistical applications: GeNorm, Normfinder, BestKeeper and Delta-Ct method. And we also performed a consensual analysis by RefFinder to make a comprehensive variability score for each reference gene. 


\section{Statistical analysis}

Continuous variables are expressed as the mean \pm the standard deviation (SD) without special instructions. A one-way analysis of variance (ANOVA) was performed to calculate whether there is statistically different among groups. Differences with a P-value $<0.05$ were considered statistically significant. All statistical analyses were performed using SPSS Statistics, version 23.0 (IBM Corp, Armonk, NY), and graphs were generated using GraphPad Prism 7 (GraphPad Software Inc., CA).

\section{Results}

\section{Expression characteristics of the reference genes}

We showed the full gene names and corresponding GeneBank accession numbers of the 21 candidate housekeeping genes in Table 1. The table 2 demonstrated the RNA concentrations at each stage of study, which ranged from $61.08-83.38 \mathrm{ng} / \mu \mathrm{l}$. No significant between-group differences were apparent in RNA content. The extracted RNA quality is shown as $260 \mathrm{~nm} / 280 \mathrm{~nm}$ ratio and $260 \mathrm{~nm} / 230 \mathrm{~nm}$ ratio assessed by Nanodrop 2000 Spectrophotometer. Both ratios were close to 2.0, indicating the high quality of the RNA samples.

For the primer sequences and the amplified product length also see Table 1. Analysis of the melting curve assays, which showed the single peak, indicated the RT-qPCR amplification had good specificity (Figure 2A). The Ct (cycle threshold) -values obtained by RT-qPCR were used to quantify the gene expression levels, with a low Ct value indicating a high gene expression level and vice versa. Figure 2B illustrated the expression in each candidate reference gene abundance across the prenatal to postnatal periods of heart development. The candidate reference genes studied presented different expression levels with the mean $\mathrm{Ct}$ value ranged from 16.16 (Gapdh) to 25.19 (Gusb). The expression of the most frequently used internal reference gene in RT-qPCR experiments, $A c t b$, revealed the unexpectedly significant variations at 7 different developmental stages of mice hearts. We found that some genes hold a more stable expression (such as Ubc, B2m, Vcp), whereas others were discrete, e.g., Ppia, Tfrc, Actb. These data illustrated the need for selection of appropriate reference genes for gene expression studies in mice hearts from different developmental stages.

For more efficient analysis of the expression pattern of those candidate reference genes at different development or maturation phases, we used the hierarchical cluster analysis (HCA) and orthogonal projections to latent structures - discriminant analysis (OPLS-DA) to globally visualize the expression classifications. Three phases can be distinguished in the gene expression features, that are (A) embryo stage, (B) first 7 days after birth, (C) 1 to 9 months after birth (Figure 3).

\section{Stability of candidate reference genes}

To assess the stability of gene expression, four different tools were used for each dataset tested in the two developmental stages addressed: GeNorm, NormFinder, Delta-Ct and BestKeeper. Table 3 demonstrated the results of the different statistical methods for analyzing the temporality expression variabilities of 21 housekeeping genes not only at earlier stages (embryonic stages). Although the scores and rank orders are not exactly the same as those reported in each of the calculation methods, the ranges of the dynamics are roughly similar in GeNorm, NormFinder, and Delta-Ct (Table 3).

GeNorm, NormFinder, and Delta-Ct, working on similar principles, held different computational approaches with BestKeeper. The stability values derived from BestKeeper based on the CV and SD values, while the former methods by comparing the average variation of a gene to all other. So, a consensual statistical analysis of the variabilities of the housekeeping genes was needed. Based on previous studies, we employed the RefFinder analysis to obtain a comprehensive evaluation of candidate reference genes by integrating all four analysis programs of the above-mentioned. The overall rank order of the most stable reference genes is shown in Table $\mathbf{3}$ for different comparisons across different developmental stages. 
We summarized these results of the overall gene stability in each dataset with different condition using the heatmap or dot plot graph to illustrate the most suitable 5 reference genes for calculating the gene expression (Figure 4). Concerning the embryo development of LV, the Ppia, RplpO, B2m, Vcp, and Gapdh were selected as the optimal reference genes when comparing the embryonic LV at 14-16 (E14-16) with LV at E17-20. In contrast, the Ppia and Gapdh were no more reliable choices for comparison of embryonic LV VS. hearts within 7 days of birth (E14-20 VS. D1-7). And the best selections of reference genes in this condition were the $V c p$, Rplpo, Ywhaz, B2m, and Hprt1 (Table 3, Figure 4). Regarding the gene expression stabilities in LV on embryonic (E14-20) and postnatal maturation stages (M1-9), the results indicated the Rplp0, Gapdh, Tbp, Vcp, Gusb should be the appropriate reference genes for RT-qPCR experiments. To make the results of RT-qPCR as accurate as possible related to the early postnatal (D1-7) and postnatal maturation stages (M1-9), Reep5, Rplp0, Polr2a, Pgk1, Rpl5 represent the best set of reference genes (Table 3, Figure 4). Finally, among the 21 reference genes evaluated for their expression variabilities in comparing all LV samples under different phases of developmental maturity, the optimal set of reference genes should be Rplpo, Tbp, Vcp, Gusb, and Rp/5. On different conditions, the appropriate set of reference genes were not the same across groups. Our results showed that following genes should not be used for normalization in RT-qPCR experiments in mice hearts: 18S, Hmbs, Ubc, Psmb4, Tfrc and Actb. And the Rplpo appeared to represent a good choice as reference gene when performing all the comparisons in the mice LV samples.

\section{Gene expression levels normalized by different reference gene}

We further verified the results by using the different internal reference genes to normalize the RT-qPCR data sets and to calculate the expression differences among groups. The most and least stable reference gene ( $R p / p 0$ and $18 S$ ) were included for RT-qPCR data analysis with the the same samples. As the results showed in Figure 5, the selection of proper internal reference gene induced very profound effects on gene expression levels. There was a large and significant difference in $V c p$ gene expression profile among the groups when $\mathrm{Rp} / \mathrm{pO}$ as reference gene for normalization (Figure 5A), however, no significance when $18 S$ used as reference gene (Figure 5B). As for the expression of $P g k 1$ in mice LVs with different stages, it is evident from Figure 5 that the statistical significance was obviously increased by the selecting of reference gene as Rplp0 rather than $18 S$.

\section{Discussions}

The previous literatures pointed out that there is no perfect internal reference gene appropriate to all situations for the quantification of target gene expression levels in RT-qPCR experiments[18]. We presented a detailed analysis of the selection of reference genes in mice LV tissues from different developmental stages in this study. And a set of the most suitable internal reference genes were proposed when normalizing the RT-qPCR data obtained from different developmental stages of mice LV tissues.

The RT-qPCR has become the most common and useful method for revealing the gene expression characteristics. Selecting an appropriate reference gene for normalization in RT-qPCR experiments was important to reduce the effect of sample heterogeneity and provide more accurate results $[13,20]$. The mechanism of heart development, which was often studied by transcriptomics, was not completely elucidated. Thus, it is crucial to identify the stable reference genes during those periods. The previous reference genes studies primarily based on myocardial tissues from adult mice or whole hearts[27]. The number of reference genes studies during heart development and maturation was relatively small. It is the limitations of previous researches that only a limited number of candidate reference genes and a small number of samples with different developmental stages were included[22,27,28]. In this study, we investigated the gene expressions of common 21 candidate housekeeping genes in 7 different developmental stages, almost throughout the whole period of the mouse lifespan. We therefore believed the results from our systematical study is likely to be more applicable and robust.

Previous investigators demonstrated that the expression of some candidate reference genes can significantly vary in different condition[22]. For example, gene 18S, Actb and Gapdh held considerably alterations of expression level depending upon developmental stages and experimental conditions[29]. It is worth mentioning that the expression of Gapdh and Actb apparently fluctuated even in normal myocardial tissues of mice. In agreement, our data also demonstrated there were altered expressions of above reference genes in developing hearts. 18S, Actb and Gapdh were the most frequently used internal reference genes in cardiac-related researches. But in our study, it is indicated the $18 S$ and Actb did not have a sufficiently stable expression. Thus, it

Page 5/20 
cannot be used as ideal reference gene in RT-qPCR experiments on LV development or maturation. The Gapdh may be redeemed as a better candidate reference gene only under some specific conditions (Regarding the gene expressions in LV at embryonic stages or comparison between embryonic (E14-20) and postnatal maturation stages (M1-9)). There is a congruence between this data with our transcriptomic data of human heart samples in different developmental stages (in-house data), suggesting the Actb and $18 S$ unsuitable as reference control in heart development studies. The results listed in Table 3 indicated the Rp/p0 have a greater gene expression stability across the sample sets. The protein, $R p / p 0$ encoding, is a component of the $60 \mathrm{~S}$ subunit and responsible for the synthesis of proteins in the cell[30]. It was the only one reference gene that cloud applied to all subgroup analysis. Based on the results, we considered the $R p / p O$ is an optimal reference gene to be used to normalize data in mice LV development or maturation.

Moreover, selecting the different reference gene as control, yielded a completely different results of the expression levels of target genes (Figure 5), which could lead to biased results and, in the worst case, wrong conclusions. This also emphasizes the importance of selecting the optimal reference gene when studying the transcriptomic signatures in heart development or maturation process.

\section{Conclusions}

Our study provides for the first time the expression stability of the commonly used reference genes in process of LV development and maturation. Through four analysis approaches (GeNorm, Normfinder, BestKeeper and the Delta-Ct) and a consensual analysis (RefFinder), we identified a set of optimal reference genes under different conditions. Our findings may be helpful in future studies to investigate the gene expression patterns and mechanism of mammalian LV development.

\section{Declarations}

\section{Acknowledgements}

We thank all the study participants.

\section{Funding Sources}

This work was supported by CAMS Innovation Fund for Medical Sciences (2016-I2M-1-015), the National Natural Science Foundation of China (81670376), the PUMC Youth Fund, and the Fundamental Research Funds for the Central Universities (3332018140)

\section{Ethical approval}

All procedures performed in studies involving animals were in accordance with the ethical standards of the institution or practice at which the studies were conducted. The study protocol was reviewed and approved by the Animal Care and Use Committee of Fuwai hospital.

Conflict of interest: None declared

Authors' contribution: JR and NNZ participated in the design of the study, performed the measurements and the statistical analysis. XJL helped in data collection and the interpretation of data. JR, XGS and JPS wrote the manuscript. All authors read and approved the manuscript.

Data availability: Data sharing are applicable to this article without restriction. 


\section{References}

1. Xin M, Olson EN, Bassel-Duby R (2013) Mending broken hearts: cardiac development as a basis for adult heart regeneration and repair. Nat Rev Mol Cell Biol 14 (8):529-541. doi:10.1038/nrm3619

2. Oh H (2017) Cell Therapy Trials in Congenital Heart Disease. Circ Res 120 (8):1353-1366. doi:10.1161/CIRCRESAHA.117.309697

3. Uosaki H, Taguchi YH (2016) Comparative Gene Expression Analysis of Mouse and Human Cardiac Maturation. Genomics Proteomics Bioinformatics 14 (4):207-215. doi:10.1016/j.gpb.2016.04.004

4. Menendez-Montes I, Escobar B, Palacios B, Gomez MJ, Izquierdo-Garcia JL, Flores L, Jimenez-Borreguero LJ, Aragones J, RuizCabello J, Torres M, Martin-Puig S (2016) Myocardial VHL-HIF Signaling Controls an Embryonic Metabolic Switch Essential for Cardiac Maturation. Dev Cell 39 (6):724-739. doi:10.1016/j.devcel.2016.11.012

5. Cohen MS, Dagincourt N, Zak V, Baffa JM, Bartz P, Dragulescu A, Dudlani G, Henderson H, Krawczeski CD, Lai WW, Levine JC, Lewis AB, McCandless RT, Ohye RG, Owens ST, Schwartz SM, Slesnick TC, Taylor CL, Frommelt PC, Pediatric Heart Network I (2018) The Impact of the Left Ventricle on Right Ventricular Function and Clinical Outcomes in Infants with Single-Right Ventricle Anomalies up to 14 Months of Age. J Am Soc Echocardiogr 31 (10):1151-1157. doi:10.1016/j.echo.2018.05.003

6. Aung N, Vargas JD, Yang C, Cabrera CP, Warren HR, Fung K, Tzanis E, Barnes MR, Rotter JI, Taylor KD, Manichaikul AW, Lima JAC, Bluemke DA, Piechnik SK, Neubauer S, Munroe PB, Petersen SE (2019) Genome-Wide Analysis of Left Ventricular ImageDerived Phenotypes Identifies Fourteen Loci Associated With Cardiac Morphogenesis and Heart Failure Development. Circulation 140 (16):1318-1330. doi:10.1161/CIRCULATIONAHA.119.041161

7. Li J, Cai SX, He Q, Zhang H, Friedberg D, Wang F, Redington AN (2018) Intravenous miR-144 reduces left ventricular remodeling after myocardial infarction. Basic Res Cardiol 113 (5):36. doi:10.1007/s00395-018-0694-x

8. Pezel T, Viallon M, Croisille P, Sebbag L, Bochaton T, Garot J, Lima JAC, Mewton N (2020) Imaging Interstitial Fibrosis, Left Ventricular Remodeling, and Function in Stage A and B Heart Failure. JACC Cardiovasc Imaging. doi:10.1016/j.jcmg.2020.05.036

9. Hashimoto H, Olson EN, Bassel-Duby R (2018) Therapeutic approaches for cardiac regeneration and repair. Nat Rev Cardiol 15 (10):585-600. doi:10.1038/s41569-018-0036-6

10. Federspiel JD, Tandon P, Wilczewski CM, Wasson L, Herring LE, Venkatesh SS, Cristea IM, Conlon FL (2019) Conservation and divergence of protein pathways in the vertebrate heart. PLoS Biol 17 (9):e3000437. doi:10.1371/journal.pbio.3000437

11. Wang Z, Cui M, Shah AM, Ye W, Tan W, Min YL, Botten GA, Shelton JM, Liu N, Bassel-Duby R, Olson EN (2019) Mechanistic basis of neonatal heart regeneration revealed by transcriptome and histone modification profiling. Proc Natl Acad Sci U S A 116 (37):18455-18465. doi:10.1073/pnas.1905824116

12. DeLaughter DM, Bick AG, Wakimoto H, McKean D, Gorham JM, Kathiriya IS, Hinson JT, Homsy J, Gray J, Pu W, Bruneau BG, Seidman JG, Seidman CE (2016) Single-Cell Resolution of Temporal Gene Expression during Heart Development. Dev Cell 39 (4):480-490. doi:10.1016/j.devcel.2016.10.001

13. Nolan T, Hands RE, Bustin SA (2006) Quantification of mRNA using real-time RT-PCR. Nat Protoc 1 (3):1559-1582. doi:10.1038/nprot.2006.236

14. Bustin SA, Benes V, Garson JA, Hellemans J, Huggett J, Kubista M, Mueller R, Nolan T, Pfaffl MW, Shipley GL, Vandesompele J, Wittwer CT (2009) The MIQE guidelines: minimum information for publication of quantitative real-time PCR experiments. Clin Chem 55 (4):611-622. doi:10.1373/clinchem.2008.112797

15. Schmittgen TD, Zakrajsek BA (2000) Effect of experimental treatment on housekeeping gene expression: validation by realtime, quantitative RT-PCR. J Biochem Biophys Methods 46 (1-2):69-81. doi:10.1016/s0165-022x(00)00129-9

16. Bustin SA, Wittwer CT (2017) MIQE: A Step Toward More Robust and Reproducible Quantitative PCR. Clin Chem 63 (9):15371538. doi:10.1373/clinchem.2016.268953

17. Lupberger J, Kreuzer KA, Baskaynak G, Peters UR, le Coutre P, Schmidt CA (2002) Quantitative analysis of beta-actin, beta-2microglobulin and porphobilinogen deaminase mRNA and their comparison as control transcripts for RT-PCR. Mol Cell Probes 16 (1):25-30. doi:10.1006/mcpr.2001.0392 
18. Huggett J, Dheda K, Bustin S, Zumla A (2005) Real-time RT-PCR normalisation; strategies and considerations. Genes Immun 6 (4):279-284. doi:10.1038/sj.gene.6364190

19. Goidin D, Mamessier A, Staquet MJ, Schmitt D, Berthier-Vergnes O (2001) Ribosomal 18S RNA prevails over glyceraldehyde-3phosphate dehydrogenase and beta-actin genes as internal standard for quantitative comparison of mRNA levels in invasive and noninvasive human melanoma cell subpopulations. Anal Biochem 295 (1):17-21. doi:10.1006/abio.2001.5171

20. Kozera B, Rapacz M (2013) Reference genes in real-time PCR. J Appl Genet 54 (4):391-406. doi:10.1007/s13353-013-0173-x

21. Vandesompele J, De Preter K, Pattyn F, Poppe B, Van Roy N, De Paepe A, Speleman F (2002) Accurate normalization of realtime quantitative RT-PCR data by geometric averaging of multiple internal control genes. Genome Biol 3 (7):RESEARCH0034. doi:10.1186/gb-2002-3-7-research0034

22. Moyses-Oliveira M, Cabral V, Gigek CO, Correa DCC, Di-Battista A, Stumpp T, Melaragno MI (2019) Search for appropriate reference genes for quantitative reverse transcription PCR studies in somite, prosencephalon and heart of early mouse embryo. Gene 710:148-155. doi:10.1016/j.gene.2019.05.042

23. Andersen CL, Jensen JL, Orntoft TF (2004) Normalization of real-time quantitative reverse transcription-PCR data: a modelbased variance estimation approach to identify genes suited for normalization, applied to bladder and colon cancer data sets. Cancer Res 64 (15):5245-5250. doi:10.1158/0008-5472.CAN-04-0496

24. Pfaffl MW, Tichopad A, Prgomet C, Neuvians TP (2004) Determination of stable housekeeping genes, differentially regulated target genes and sample integrity: BestKeeper-Excel-based tool using pair-wise correlations. Biotechnol Lett 26 (6):509-515. doi:10.1023/b:bile.0000019559.84305.47

25. Silver N, Best S, Jiang J, Thein SL (2006) Selection of housekeeping genes for gene expression studies in human reticulocytes using real-time PCR. BMC Mol Biol 7:33. doi:10.1186/1471-2199-7-33

26. Xie $F$, Xiao $P$, Chen $D, X u L$, Zhang $B$ (2012) miRDeepFinder: a miRNA analysis tool for deep sequencing of plant small RNAs. Plant Mol Biol. doi:10.1007/s11103-012-9885-2

27. Ruiz-Villalba A, Mattiotti A, Gunst QD, Cano-Ballesteros S, van den Hoff MJ, Ruijter JM (2017) Reference genes for gene expression studies in the mouse heart. Sci Rep 7 (1):24. doi:10.1038/s41598-017-00043-9

28. Medrano G, Guan P, Barlow-Anacker AJ, Gosain A (2017) Comprehensive selection of reference genes for quantitative RT-PCR analysis of murine extramedullary hematopoiesis during development. PLoS One 12 (7):e0181881. doi:10.1371/journal.pone.0181881

29. Li M, Rao M, Chen K, Zhou J, Song J (2017) Selection of reference genes for gene expression studies in heart failure for left and right ventricles. Gene 620:30-35. doi:10.1016/j.gene.2017.04.006

30. Wang CH, Wang LK, Wu CC, Chen ML, Lee MC, Lin YY, Tsai FM (2019) The Ribosomal Protein RPLP0 Mediates PLAAT4induced Cell Cycle Arrest and Cell Apoptosis. Cell Biochem Biophys 77 (3):253-260. doi:10.1007/s12013-019-00876-3

\section{Tables}

Table 1. Candidate reference genes and primer sequences 


\begin{tabular}{|c|c|c|c|c|}
\hline $\begin{array}{l}\text { Gene } \\
\text { symbol }\end{array}$ & Gene Name & $\begin{array}{l}\text { GenBank } \\
\text { Accession }\end{array}$ & $\begin{array}{l}\text { 5'-Primer Sequences } \\
\text { (Forward/Reverse)-3' }\end{array}$ & $\begin{array}{l}\text { Product } \\
\text { size }\end{array}$ \\
\hline Actb & Actin beta & NM_007393 & $\begin{array}{l}\text { GGCTGTATTCCCCTCCATCG / } \\
\text { CCAGTTGGTAACAATGCCATGT }\end{array}$ & $154 \mathrm{bp}$ \\
\hline Gapdh & Glyceraldehyde-3-phosphate dehydrogenase & NM_001289726 & $\begin{array}{l}\text { AGGTCGGTGTGAACGGATTTG / } \\
\text { TGTAGACCATGTAGTTGAGGTCA }\end{array}$ & $123 \mathrm{bp}$ \\
\hline Reep5 & Receptor accessory protein 5 & NM_007874 & $\begin{array}{l}\text { GGTTCCTGCACGAGAAGAACT / } \\
\text { GAGAGAGGCTCCATAACCGAA }\end{array}$ & $140 \mathrm{bp}$ \\
\hline Rpl5 & Ribosomal protein L5 & NM_016980 & $\begin{array}{l}\text { TTGGTGATCCAGGACAAGAATAA / } \\
\text { GCACAGACGATCATATCCCC }\end{array}$ & $125 \mathrm{bp}$ \\
\hline Psmb4 & Proteasome subunit beta 4 & NM_008945 & $\begin{array}{l}\text { ATGGAAGCGTTTTGGGAGTCA / } \\
\text { GTTCTGGGTCCGAGTGATGG }\end{array}$ & $144 \mathrm{bp}$ \\
\hline Vcp & Valosin containing protein & NM_009503 & $\begin{array}{l}\text { GCTTGTAAACTGGCCATTCG / } \\
\text { GATCTCAGGCACTGGATCGT }\end{array}$ & $114 \mathrm{bp}$ \\
\hline B2m & Beta-2-microglobulin & NM_009735 & $\begin{array}{l}\text { TTCTGGTGCTTGTCTCACTGA / } \\
\text { CAGTATGTTCGGCTTCCCATTC }\end{array}$ & $104 \mathrm{bp}$ \\
\hline Gusb & Glucuronidase beta & NM_010368 & $\begin{array}{l}\text { GGCTGGTGACCTACTGGATTT / } \\
\text { GGCACTGGGAACCTGAAGT }\end{array}$ & $131 \mathrm{bp}$ \\
\hline Hmbs & Hydroxymethylbilane synthase & NM_001110251 & $\begin{array}{l}\text { AAGGGCTTTTCTGAGGCACC / } \\
\text { AGTTGCCCATCTTTCATCACTG }\end{array}$ & 78 bp \\
\hline Hprt1 & Hypoxanthine phosphoribosyltransferase 1 & NM_013556 & $\begin{array}{l}\text { GGTTAAGCAGTACAGCCCCA / } \\
\text { GGCCTGTATCCAACACTTCG }\end{array}$ & $81 \mathrm{bp}$ \\
\hline Ipo8 & Importin 8 & NM_001081113 & $\begin{array}{l}\text { ACGTGACAGTAGATACCAACGC / } \\
\text { GCATAGCACTCGGCATCTTCT }\end{array}$ & $115 \mathrm{bp}$ \\
\hline Pgk1 & Phosphoglycerate kinase 1 & NM_008828 & $\begin{array}{l}\text { ATGTCGCTTTCCAACAAGCTG / } \\
\text { GCTCCATTGTCCAAGCAGAAT }\end{array}$ & $164 \mathrm{bp}$ \\
\hline Polr2a & RNA polymerase II subunit A & NM_001291068 & $\begin{array}{l}\text { AAATACCCAGAAACAACGGAGG / } \\
\text { CCAGTCCGCTCAATCACCC }\end{array}$ & $83 \mathrm{bp}$ \\
\hline Ppia & Peptidylprolyl isomerase A & NM_008907 & $\begin{array}{l}\text { GAGCTGTTTGCAGACAAAGTTC / } \\
\text { CCCTGGCACATGAATCCTGG }\end{array}$ & $125 \mathrm{bp}$ \\
\hline Rplp0 & Ribosomal protein lateral stalk subunit P0 & NM_007475 & $\begin{array}{l}\text { AGATTCGGGATATGCTGTTGGC / } \\
\text { TCGGGTCCTAGACCAGTGTTC }\end{array}$ & $109 \mathrm{bp}$ \\
\hline Tbp & TATA box binding protein & NM_013684 & $\begin{array}{l}\text { GTGGGGAGCTGTGATGTGA / } \\
\text { TCCAGGAAATAATTCTGGCTCA }\end{array}$ & $96 \mathrm{bp}$ \\
\hline Tfrc & Transferrin receptor & NM_011638 & $\begin{array}{l}\text { GTTTCTGCCAGCCCCTTATTAT / } \\
\text { GCAAGGAAAGGATATGCAGCA }\end{array}$ & $152 \mathrm{bp}$ \\
\hline Ubc & Ubiquitin C & NM_019639 & $\begin{array}{l}\text { GAGGTGGCATGCAGATCTTT / } \\
\text { CCCTCCTTGTCCTGGATCTT }\end{array}$ & $112 \mathrm{bp}$ \\
\hline Ywhaz & $\begin{array}{l}\text { Tyrosine 3-monooxygenase/tryptophan 5- } \\
\text { monooxygenase activation protein zeta }\end{array}$ & NM_011740 & $\begin{array}{l}\text { GAAAAGTTCTTGATCCCCAATGC / } \\
\text { TGTGACTGGTCCACAATTCCTT }\end{array}$ & $134 \mathrm{bp}$ \\
\hline $18 S$ & eukaryotic $18 \mathrm{~S}$ ribosomal RNA & NR_003278 & $\begin{array}{l}\text { CTCAACACGGGAAACCTCAC / } \\
\text { CGCTCCACCAACTAAGAACG }\end{array}$ & $110 \mathrm{bp}$ \\
\hline Sdha & $\begin{array}{l}\text { succinate dehydrogenase complex } \\
\text { flavoprotein subunit A }\end{array}$ & NM_023281 & $\begin{array}{l}\text { GGAACACTCCAAAAACAGACCT / } \\
\text { CCACCACTGGGTATTGAGTAGAA }\end{array}$ & $106 \mathrm{bp}$ \\
\hline
\end{tabular}

Table 2. The quality of RNA samples isolated from left ventricles 


\begin{tabular}{|llllllll|}
\hline & \multirow{2}{*}{ N } & RNA content $(\mathrm{ng} / \mathrm{ul})$ & A260 (Abs) & \multicolumn{2}{l}{$260 \mathrm{~nm} / 280 \mathrm{~nm}$ ratio } & \multicolumn{2}{c|}{ 260nm/230 $\mathrm{nm}$ ratio } \\
\cline { 7 - 8 } & & & & mean & SD & mean & SD \\
\hline E 14 16 & 4 & $67.65 \pm 2.92$ & $1.70 \pm 0.07$ & 1.97 & 0.03 & 2.13 & 0.09 \\
\hline E 17-20 & 5 & $66.98 \pm 2.40$ & $1.67 \pm 0.06$ & 1.99 & 0.03 & 2.08 & 0.13 \\
\hline D 1 3 & 6 & $61.08 \pm 4.56$ & $1.53 \pm 0.11$ & 2.02 & 0.01 & 2.09 & 0.07 \\
\hline D 4 7 & 5 & $69.12 \pm 9.59$ & $1.73 \pm 0.24$ & 2.03 & 0.01 & 2.05 & 0.14 \\
\hline M 1 2 & 5 & $83.38 \pm 22.58$ & $2.08 \pm 0.56$ & 2.05 & 0.02 & 2.21 & 0.05 \\
\hline M 3 5 & 7 & $65.57 \pm 4.78$ & $1.64 \pm 0.12$ & 2.08 & 0.02 & 1.97 & 0.17 \\
\hline M 6 9 & 6 & $66.17 \pm 3.67$ & $1.65 \pm 0.09$ & 2.06 & 0.02 & 2.16 & 0.08 \\
\hline
\end{tabular}

$\mathrm{E}=$ embryonic day; $\mathrm{D}=$ postnatal day; $\mathrm{M}=$ postnatal month; $\mathrm{N}=$ sample size; $\mathrm{SD}=$ standard deviation

Table 3. Reference gene expression variability and rankings 


\begin{tabular}{|c|c|c|c|c|c|c|c|c|c|}
\hline \multicolumn{2}{|l|}{ GeNorm } & \multicolumn{2}{|c|}{ NormFinder } & \multicolumn{2}{|c|}{ BestKeeper } & \multicolumn{2}{|c|}{ Delta CT } & \multicolumn{2}{|c|}{ RefFinder } \\
\hline $\begin{array}{l}\text { Gene } \\
\text { name }\end{array}$ & $\begin{array}{l}\text { Stability } \\
\text { value }\end{array}$ & $\begin{array}{l}\text { Gene } \\
\text { name }\end{array}$ & $\begin{array}{l}\text { Stability } \\
\text { value }\end{array}$ & $\begin{array}{l}\text { Gene } \\
\text { name }\end{array}$ & $\begin{array}{l}\text { std } \\
\text { dev }\end{array}$ & $\begin{array}{l}\text { Gene } \\
\text { name }\end{array}$ & STDEV & $\begin{array}{l}\text { Gene } \\
\text { name }\end{array}$ & $\begin{array}{l}\text { Geomean } \\
\text { of } \\
\text { ranking } \\
\text { values }\end{array}$ \\
\hline \multicolumn{10}{|c|}{$\begin{array}{l}\text { E 14 16/E } \\
17 \sim 20(n=4 / 5)\end{array}$} \\
\hline Ppia & 0.064 & Ppia & 0.032 & Hprt1 & 0.24 & Ppia & 0.46 & Ppia & 1.57 \\
\hline Rplp0 & 0.064 & B2m & 0.039 & Reep5 & 0.26 & B2m & 0.46 & Rplp0 & 2.82 \\
\hline Vcp & 0.081 & Rplp0 & 0.099 & Gapdh & 0.27 & Rplp0 & 0.47 & B2m & 3.36 \\
\hline B2m & 0.093 & Vcp & 0.13 & $V_{c p}$ & 0.35 & Vcp & 0.47 & Vcp & 3.94 \\
\hline Tbp & 0.109 & Ywhaz & 0.138 & Ubc & 0.35 & Tbp & 0.5 & Gapdh & 6.42 \\
\hline Rpl5 & 0.12 & Tfrc & 0.161 & Ppia & 0.4 & Ywhaz & 0.5 & Hprt1 & 6.57 \\
\hline Gapdh & 0.14 & Rpl5 & 0.185 & Rplp0 & 0.41 & Rpl5 & 0.5 & Tbp & 6.69 \\
\hline Ywhaz & 0.174 & Tbp & 0.186 & B2m & 0.44 & Tfrc & 0.51 & Rpl5 & 7.17 \\
\hline Tfrc & 0.206 & Gapdh & 0.258 & Rpl5 & 0.44 & Gapdh & 0.52 & Ywhaz & 7.33 \\
\hline Ubc & 0.233 & Ubc & 0.263 & Tbp & 0.45 & Ubc & 0.55 & Ubc & 7.95 \\
\hline Hprt1 & 0.254 & Gusb & 0.317 & Tfrc & 0.47 & Gusb & 0.57 & Tfrc & 8.3 \\
\hline Gusb & 0.284 & Actb & 0.334 & Ywhaz & 0.57 & Actb & 0.59 & Reep5 & 9.36 \\
\hline Actb & 0.303 & Hprt1 & 0.394 & Sdha & 0.62 & Hprt1 & 0.61 & Gusb & 12.35 \\
\hline Ipo8 & 0.341 & Ipo8 & 0.487 & Ipo8 & 0.67 & Ipo8 & 0.68 & Actb & 13.55 \\
\hline Reep5 & 0.378 & Sdha & 0.573 & Hmbs & 0.68 & Sdha & 0.75 & Ipo8 & 14 \\
\hline Sdha & 0.409 & Reep5 & 0.593 & Gusb & 0.7 & Reep5 & 0.76 & Sdha & 14.71 \\
\hline Hmbs & 0.462 & Hmbs & 0.806 & Pgk1 & 0.7 & $\mathrm{Hmbs}$ & 0.94 & Hmbs & 16.48 \\
\hline Psmb4 & 0.51 & Psmb4 & 0.808 & Actb & 0.74 & Psmb4 & 0.95 & Psmb4 & 18.24 \\
\hline Polr2a & 0.552 & Polr2a & 0.857 & Psmb4 & 0.85 & Polr2a & 0.95 & Polr2a & 19.25 \\
\hline $18 \mathrm{~S}$ & 0.605 & $18 \mathrm{~S}$ & 1.063 & Polr2a & 1.01 & $18 \mathrm{~S}$ & 1.14 & Pgk1 & 19.92 \\
\hline Pgk1 & 0.68 & Pgk1 & 1.351 & $18 \mathrm{~S}$ & 1.19 & Pgk1 & 1.4 & $18 \mathrm{~S}$ & 20.25 \\
\hline \multicolumn{10}{|c|}{$\begin{array}{l}\text { E 14 20/ } \\
\text { D1 7(n=9/11) }\end{array}$} \\
\hline Vcp & 0.103 & Vcp & 0.075 & Hprt1 & 0.23 & Vcp & 0.46 & Vcp & 1.19 \\
\hline Rplp0 & 0.103 & Rplp0 & 0.097 & $V_{c p}$ & 0.25 & Rplp0 & 0.47 & Rplp0 & 2.11 \\
\hline Ywhaz & 0.175 & Ywhaz & 0.162 & $\mathrm{~B} 2 \mathrm{~m}$ & 0.28 & Ywhaz & 0.48 & Ywhaz & 3.71 \\
\hline B2m & 0.21 & B2m & 0.194 & Tbp & 0.28 & Gusb & 0.49 & B2m & 4.23 \\
\hline Tbp & 0.225 & Gusb & 0.2 & Rplp0 & 0.31 & $\mathrm{~B} 2 \mathrm{~m}$ & 0.49 & Hprt1 & 5.2 \\
\hline Gusb & 0.243 & Ppia & 0.218 & Ppia & 0.36 & Ppia & 0.51 & Tbp & 5.57 \\
\hline Actb & 0.255 & Actb & 0.242 & Ywhaz & 0.37 & Actb & 0.52 & Gusb & 6.03 \\
\hline Ppia & 0.277 & Tbp & 0.256 & Reep5 & 0.39 & Tbp & 0.52 & Ppia & 6.45 \\
\hline Hprt1 & 0.292 & Hprt1 & 0.261 & Ipo8 & 0.39 & Hprt1 & 0.54 & Actb & 8.47 \\
\hline
\end{tabular}




\begin{tabular}{|c|c|c|c|c|c|c|c|c|c|}
\hline Rpl5 & 0.306 & Tfrc & 0.285 & Tfrc & 0.39 & Tfrc & 0.55 & Tfrc & 10.24 \\
\hline Tfrc & 0.326 & Rpl5 & 0.32 & Gusb & 0.4 & Ipo8 & 0.56 & Ipo8 & 10.93 \\
\hline Ipo8 & 0.341 & Ipo8 & 0.321 & Sdha & 0.43 & Rpl5 & 0.56 & Rpl5 & 11.45 \\
\hline Polr2a & 0.38 & Polr2a & 0.576 & Ubc & 0.44 & Polr2a & 0.71 & Sdha & 13.71 \\
\hline Sdha & 0.419 & Gapdh & 0.604 & Actb & 0.44 & Sdha & 0.75 & Polr2a & 13.9 \\
\hline Ubc & 0.45 & Sdha & 0.62 & Rpl5 & 0.44 & Gapdh & 0.75 & Reep5 & 14.07 \\
\hline Reep5 & 0.477 & Ubc & 0.643 & Hmbs & 0.53 & Ubc & 0.77 & Ubc & 15.23 \\
\hline Gapdh & 0.507 & Psmb4 & 0.655 & Polr2a & 0.55 & Reep5 & 0.79 & Gapdh & 15.92 \\
\hline Psmb4 & 0.535 & Reep5 & 0.656 & Psmb4 & 0.65 & Psmb4 & 0.8 & Psmb4 & 17.99 \\
\hline Hmbs & 0.561 & Hmbs & 0.67 & Gapdh & 0.65 & Hmbs & 0.82 & Hmbs & 18.2 \\
\hline $18 \mathrm{~S}$ & 0.586 & $18 \mathrm{~S}$ & 0.733 & $18 \mathrm{~S}$ & 0.73 & $18 \mathrm{~S}$ & 0.85 & $18 \mathrm{~S}$ & 20 \\
\hline Pgk1 & 0.65 & Pgk1 & 1.197 & Pgk1 & 0.88 & Pgk1 & 1.26 & Pgk1 & 21 \\
\hline \multicolumn{10}{|l|}{$\begin{array}{l}\text { E 14 20/ } \\
\text { M1 9 } \\
(n=9 / 18)\end{array}$} \\
\hline Rplp0 & 0.236 & Rplp0 & 0.068 & Reep5 & 0.35 & Rplp0 & 0.64 & Rplp0 & 1.78 \\
\hline Gapdh & 0.236 & Tbp & 0.07 & Ubc & 0.35 & Tbp & 0.65 & Gapdh & 3.22 \\
\hline Rpl5 & 0.268 & Gapdh & 0.248 & B2m & 0.39 & Gapdh & 0.69 & Tbp & 3.44 \\
\hline Hprt1 & 0.278 & Vcp & 0.276 & Vcp & 0.43 & Gusb & 0.69 & Vcp & 5.26 \\
\hline Tbp & 0.295 & Gusb & 0.281 & Sdha & 0.49 & Rpl5 & 0.71 & Gusb & 6.03 \\
\hline Gusb & 0.319 & Rpl5 & 0.371 & Ipo8 & 0.51 & $V_{c p}$ & 0.71 & Rpl5 & 6.06 \\
\hline Ywhaz & 0.35 & Hprt1 & 0.409 & Tbp & 0.64 & Hprt1 & 0.73 & Hprt1 & 7.24 \\
\hline Vcp & 0.393 & Ipo8 & 0.438 & Hmbs & 0.69 & Ipo8 & 0.78 & Ipo8 & 8.06 \\
\hline Hmbs & 0.433 & Hmbs & 0.455 & Polr2a & 0.71 & Ywhaz & 0.79 & Reep5 & 8.44 \\
\hline Psmb4 & 0.47 & Polr2a & 0.514 & Rplp0 & 0.76 & Hmbs & 0.8 & Hmbs & 8.97 \\
\hline Ipo8 & 0.506 & Psmb4 & 0.531 & Gusb & 0.81 & Polr2a & 0.83 & Ubc & 9.46 \\
\hline Polr2a & 0.533 & Ywhaz & 0.545 & Gapdh & 0.84 & Psmb4 & 0.84 & Polr2a & 10.44 \\
\hline Actb & 0.572 & Reep5 & 0.669 & Psmb4 & 0.86 & Reep5 & 0.91 & Ywhaz & 10.65 \\
\hline Ppia & 0.609 & $18 \mathrm{~S}$ & 0.77 & Hprt1 & 0.95 & Actb & 0.98 & Psmb4 & 11.45 \\
\hline Reep5 & 0.649 & Actb & 0.835 & Rpl5 & 1 & $18 \mathrm{~S}$ & 1.01 & B2m & 11.65 \\
\hline $18 \mathrm{~S}$ & 0.683 & Ppia & 0.936 & $18 \mathrm{~S}$ & 1.05 & Ppia & 1.06 & Sdha & 14.67 \\
\hline Pgk1 & 0.723 & Tfrc & 1.008 & Ywhaz & 1.07 & $\mathrm{~B} 2 \mathrm{~m}$ & 1.15 & Actb & 15.09 \\
\hline Tfrc & 0.762 & Pgk1 & 1.014 & Pgk1 & 1.21 & Pgk1 & 1.16 & $18 \mathrm{~S}$ & 15.23 \\
\hline B2m & 0.812 & B2m & 1.03 & Actb & 1.29 & Tfrc & 1.18 & Ppia & 16.36 \\
\hline Ubc & 0.855 & Ubc & 1.101 & Ppia & 1.42 & Ubc & 1.2 & Pgk1 & 17.74 \\
\hline Sdha & 0.893 & Sdha & 1.155 & Tfrc & 1.51 & Sdha & 1.26 & Tfrc & 18.69 \\
\hline $\begin{array}{l}\text { D 1 7/ M1 9 } \\
(n=11 / 18)\end{array}$ & & & & & & & & & \\
\hline
\end{tabular}




\begin{tabular}{|c|c|c|c|c|c|c|c|c|c|}
\hline Reep5 & 0.174 & Rplp0 & 0.075 & Ubc & 0.18 & Rplp0 & 0.48 & Reep5 & 2.38 \\
\hline Polr2a & 0.174 & Reep5 & 0.085 & B2m & 0.32 & Reep5 & 0.48 & Rplp0 & 2.58 \\
\hline Pgk1 & 0.195 & Polr2a & 0.106 & Sdha & 0.32 & Polr2a & 0.48 & Polr2a & 2.82 \\
\hline Rplp0 & 0.211 & Rpl5 & 0.126 & Ipo8 & 0.33 & Rpl5 & 0.5 & Pgk1 & 5.23 \\
\hline Rpl5 & 0.223 & Pgk1 & 0.165 & $V_{c p}$ & 0.34 & Pgk1 & 0.51 & Rpl5 & 5.89 \\
\hline Tbp & 0.248 & Tbp & 0.212 & Gapdh & 0.5 & Tbp & 0.52 & Vcp & 6.88 \\
\hline Gusb & 0.263 & Gusb & 0.249 & Polr2a & 0.51 & Vcp & 0.53 & Tbp & 7.14 \\
\hline Vcp & 0.284 & Vcp & 0.283 & Reep5 & 0.52 & Gusb & 0.54 & Ipo8 & 7.35 \\
\hline Ipo8 & 0.299 & Ipo8 & 0.32 & Hmbs & 0.52 & Ipo8 & 0.55 & Ubc & 8 \\
\hline Hprt1 & 0.321 & Hprt1 & 0.33 & Pgk1 & 0.61 & Hprt1 & 0.58 & Gusb & 8.9 \\
\hline Hmbs & 0.345 & Hmbs & 0.395 & Rplp0 & 0.62 & Hmbs & 0.62 & Sdha & 9.65 \\
\hline Psmb4 & 0.37 & Psmb4 & 0.41 & Tbp & 0.64 & Gapdh & 0.64 & Hmbs & 10.46 \\
\hline Gapdh & 0.39 & Gapdh & 0.455 & $18 \mathrm{~S}$ & 0.66 & Psmb4 & 0.64 & Gapdh & 10.5 \\
\hline $18 \mathrm{~S}$ & 0.422 & Ywhaz & 0.55 & Psmb4 & 0.67 & Ywhaz & 0.7 & Hprt1 & 11.42 \\
\hline Sdha & 0.452 & $18 \mathrm{~S}$ & 0.551 & Rpl5 & 0.71 & $18 \mathrm{~S}$ & 0.73 & B2m & 11.82 \\
\hline Ubc & 0.475 & Ubc & 0.643 & Gusb & 0.73 & Ubc & 0.76 & Psmb4 & 12.72 \\
\hline Ywhaz & 0.5 & Sdha & 0.665 & Hprt1 & 0.84 & Sdha & 0.77 & $18 \mathrm{~S}$ & 14.23 \\
\hline B2m & 0.536 & Ppia & 0.755 & Ywhaz & 0.98 & Ppia & 0.85 & Ywhaz & 15.65 \\
\hline Ppia & 0.572 & B2m & 0.888 & Ppia & 1.18 & B2m & 0.95 & Ppia & 18.49 \\
\hline Actb & 0.615 & Actb & 0.929 & Actb & 1.32 & Actb & 0.99 & Actb & 20 \\
\hline Tfrc & 0.664 & Tfrc & 1.051 & Tfrc & 1.47 & Tfrc & 1.14 & Tfrc & 21 \\
\hline \multicolumn{10}{|c|}{$\begin{array}{l}\text { D 1 3/ D 4 7 } \\
(n=6 / 5)\end{array}$} \\
\hline$V_{c p}$ & 0.056 & Gusb & 0.072 & Ipo8 & 0.13 & $V_{c p}$ & 0.23 & Vcp & 1.78 \\
\hline Rplp0 & 0.056 & Vcp & 0.08 & B2m & 0.14 & Gusb & 0.23 & Rplp0 & 2.45 \\
\hline Gusb & 0.074 & Rpl5 & 0.088 & Rplp0 & 0.14 & Rplp0 & 0.23 & Gusb & 2.45 \\
\hline B2m & 0.095 & Rplp0 & 0.09 & Tbp & 0.14 & Rpl5 & 0.24 & Ipo8 & 4.6 \\
\hline Polr2a & 0.112 & Ppia & 0.094 & Gusb & 0.15 & Ppia & 0.24 & Rpl5 & 4.9 \\
\hline Rpl5 & 0.125 & Sdha & 0.099 & Vcp & 0.15 & Sdha & 0.24 & B2m & 6.45 \\
\hline Sdha & 0.133 & Ipo8 & 0.102 & Hprt1 & 0.17 & Hprt1 & 0.25 & Sdha & 7.09 \\
\hline Ipo8 & 0.141 & Hprt1 & 0.107 & Polr2a & 0.17 & Ipo8 & 0.25 & Ppia & 7.24 \\
\hline Hprt1 & 0.149 & Actb & 0.124 & Sdha & 0.17 & B2m & 0.25 & Hprt1 & 7.71 \\
\hline Ppia & 0.153 & Reep5 & 0.125 & Rpl5 & 0.17 & Polr2a & 0.26 & Polr2a & 8.39 \\
\hline Reep5 & 0.157 & Polr2a & 0.128 & Ppia & 0.18 & Reep5 & 0.26 & Tbp & 9.06 \\
\hline Actb & 0.161 & B2m & 0.133 & Ywhaz & 0.18 & Actb & 0.26 & Reep5 & 11.2 \\
\hline Ywhaz & 0.169 & Ywhaz & 0.206 & Reep5 & 0.19 & Ywhaz & 0.29 & Actb & 11.81 \\
\hline Pgk1 & 0.182 & Pgk1 & 0.217 & Ubc & 0.19 & Pgk1 & 0.31 & Ywhaz & 12.74 \\
\hline
\end{tabular}




\begin{tabular}{|c|c|c|c|c|c|c|c|c|c|}
\hline Tbp & 0.196 & Tbp & 0.269 & Actb & 0.21 & Tbp & 0.34 & Pgk1 & 14.48 \\
\hline Gapdh & 0.21 & Gapdh & 0.289 & Pgk1 & 0.26 & Gapdh & 0.36 & Ubc & 16.66 \\
\hline Ubc & 0.225 & $18 \mathrm{~S}$ & 0.313 & $18 \mathrm{~S}$ & 0.26 & $18 \mathrm{~S}$ & 0.38 & Gapdh & 16.7 \\
\hline $18 \mathrm{~S}$ & 0.237 & Ubc & 0.325 & Tfrc & 0.33 & Ubc & 0.38 & $18 \mathrm{~S}$ & 17.24 \\
\hline Tfrc & 0.253 & Tfrc & 0.345 & Gapdh & 0.35 & Tfrc & 0.41 & Tfrc & 18.74 \\
\hline Psmb4 & 0.283 & Psmb4 & 0.529 & Hmbs & 0.36 & Psmb4 & 0.57 & Psmb4 & 20.25 \\
\hline $\mathrm{Hmbs}$ & 0.314 & Hmbs & 0.575 & Psmb4 & 0.4 & $\mathrm{Hmbs}$ & 0.61 & Hmbs & 20.75 \\
\hline \multicolumn{10}{|c|}{$M 1 \sim 9(n=18)$} \\
\hline Polr2a & 0.102 & Reep5 & 0.039 & Ubc & 0.17 & Reep5 & 0.28 & Reep5 & 2.45 \\
\hline Sdha & 0.102 & Sdha & 0.047 & $V_{c p}$ & 0.24 & Sdha & 0.28 & Sdha & 2.69 \\
\hline Reep5 & 0.118 & Ppia & 0.073 & $\mathrm{~B} 2 \mathrm{~m}$ & 0.25 & Ppia & 0.29 & Polr2a & 3.76 \\
\hline Ywhaz & 0.13 & Rplp0 & 0.079 & Ipo8 & 0.31 & Rplp0 & 0.29 & Ppia & 5.3 \\
\hline Gusb & 0.137 & Polr2a & 0.108 & Gusb & 0.33 & Polr2a & 0.29 & Rplp0 & 6.16 \\
\hline Ipo8 & 0.14 & Ywhaz & 0.115 & Tbp & 0.33 & Tbp & 0.29 & Ywhaz & 6.24 \\
\hline Tbp & 0.143 & Hmbs & 0.119 & $\mathrm{Hmbs}$ & 0.35 & Ywhaz & 0.3 & Gusb & 6.51 \\
\hline Ppia & 0.149 & Tbp & 0.123 & Polr2a & 0.36 & Gusb & 0.3 & Tbp & 6.7 \\
\hline Rplp0 & 0.152 & Gusb & 0.137 & Ywhaz & 0.37 & Hmbs & 0.3 & Ipo8 & 7.33 \\
\hline $\mathrm{Hmbs}$ & 0.155 & Hprt1 & 0.144 & Rplp0 & 0.38 & Ipo8 & 0.31 & Hmbs & 8.15 \\
\hline Hprt1 & 0.164 & Pgk1 & 0.152 & Reep5 & 0.39 & Hprt1 & 0.32 & Vcp & 8.9 \\
\hline Pgk1 & 0.174 & Ipo8 & 0.164 & Ppia & 0.39 & Pgk1 & 0.34 & Ubc & 9.1 \\
\hline Rpl5 & 0.183 & Rpl5 & 0.166 & Sdha & 0.41 & Rpl5 & 0.34 & Hprt1 & 11.41 \\
\hline$V_{c p}$ & 0.192 & Actb & 0.236 & Hprt1 & 0.43 & Vcp & 0.35 & $\mathrm{~B} 2 \mathrm{~m}$ & 11.5 \\
\hline Actb & 0.203 & Gapdh & 0.242 & Actb & 0.43 & Actb & 0.38 & Pgk1 & 12.81 \\
\hline Gapdh & 0.215 & $V_{c p}$ & 0.245 & Rpl5 & 0.48 & Gapdh & 0.39 & Rpl5 & 13.69 \\
\hline Psmb4 & 0.237 & Psmb4 & 0.356 & Pgk1 & 0.49 & Psmb4 & 0.47 & Actb & 14.74 \\
\hline B2m & 0.261 & B2m & 0.471 & Psmb4 & 0.52 & B2m & 0.52 & Gapdh & 16.44 \\
\hline Ubc & 0.281 & Ubc & 0.473 & Gapdh & 0.54 & Ubc & 0.53 & Psmb4 & 17.24 \\
\hline $18 \mathrm{~S}$ & 0.321 & $18 \mathrm{~S}$ & 0.625 & $18 \mathrm{~S}$ & 0.72 & $18 \mathrm{~S}$ & 0.7 & $18 \mathrm{~S}$ & 20 \\
\hline Tfrc & 0.402 & Tfrc & 1.15 & Tfrc & 1.25 & Tfrc & 1.17 & Tfrc & 21 \\
\hline \multicolumn{10}{|c|}{ Total $(n=38)$} \\
\hline Rplp0 & 0.221 & Rplp0 & 0.044 & Ubc & 0.33 & Rplp0 & 0.61 & Rplp0 & 1.78 \\
\hline Tbp & 0.221 & Tbp & 0.188 & $\mathrm{~B} 2 \mathrm{~m}$ & 0.37 & Tbp & 0.64 & Tbp & 2.45 \\
\hline Gusb & 0.268 & Vcp & 0.267 & Vcp & 0.39 & Gusb & 0.66 & Vcp & 3.83 \\
\hline Rpl5 & 0.299 & Gusb & 0.277 & Sdha & 0.4 & Vcp & 0.67 & Gusb & 4.65 \\
\hline Hprt1 & 0.321 & Rpl5 & 0.312 & Ipo8 & 0.43 & Rpl5 & 0.67 & Rpl5 & 6.12 \\
\hline$V_{c p}$ & 0.373 & Hprt1 & 0.389 & Reep5 & 0.46 & Hprt1 & 0.71 & Ipo8 & 6.44 \\
\hline
\end{tabular}




\begin{tabular}{|llllllllll|}
\hline Ipo8 & 0.429 & Ipo8 & 0.405 & Hmbs & 0.64 & Ipo8 & 0.73 & Hprt1 & 7.33 \\
\hline Polr2a & 0.457 & Polr2a & 0.432 & Polr2a & 0.65 & Polr2a & 0.75 & Polr2a & 8 \\
\hline Gapdh & 0.486 & Gapdh & 0.476 & Tbp & 0.66 & Gapdh & 0.78 & Ubc & 8.98 \\
\hline Hmbs & 0.51 & Hmbs & 0.502 & Rplp0 & 0.68 & Ywhaz & 0.79 & Hmbs & 9.37 \\
\hline Ywhaz & 0.533 & Psmb4 & 0.52 & Gapdh & 0.69 & Hmbs & 0.8 & Gapdh & 9.46 \\
\hline Psmb4 & 0.552 & Ywhaz & 0.568 & Psmb4 & 0.77 & Psmb4 & 0.81 & Reep5 & 10.72 \\
\hline Reep5 & 0.58 & Reep5 & 0.57 & Gusb & 0.81 & Reep5 & 0.83 & B2m & 11.1 \\
\hline 18S & 0.61 & 18S & 0.683 & Rpl5 & 0.82 & 18S & 0.92 & Psmb4 & 11.74 \\
\hline Ppia & 0.651 & Ppia & 0.882 & 18S & 0.85 & Ppia & 1.01 & Ywhaz & 12.42 \\
\hline Actb & 0.682 & Pgk1 & 0.902 & Hprt1 & 0.91 & Actb & 1.04 & Sdha & 13.87 \\
\hline Pgk1 & 0.716 & Actb & 0.918 & Pgk1 & 0.93 & Pgk1 & 1.07 & $18 S$ & 14.24 \\
\hline Tfrc & 0.748 & Tfrc & 0.932 & Ywhaz & 1.11 & Ubc & 1.07 & Ppia & 16.12 \\
\hline Ubc & 0.79 & Ubc & 0.946 & Tfrc & 1.34 & B2m & 1.09 & Pgk1 & 16.74 \\
\hline B2m & 0.823 & B2m & 0.973 & Ppia & 1.4 & Tfrc & 1.1 & Actb & 17.39 \\
\hline Sdha & 0.851 & Sdha & 1.004 & Actb & 1.42 & Sdha & 1.12 & Tfrc & 18.73 \\
\hline
\end{tabular}

\section{Figures}




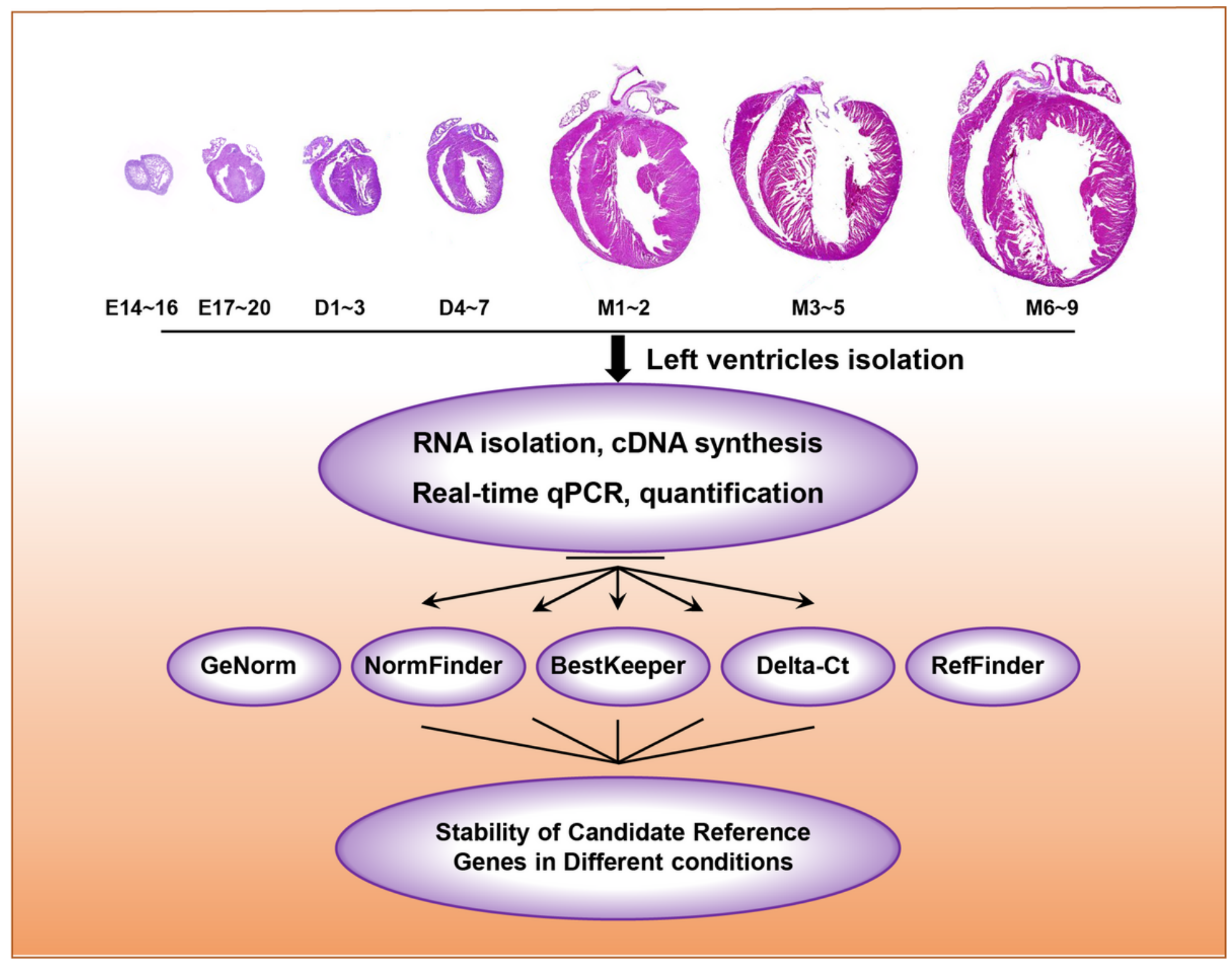

Figure 1

Flow chart of the study design. Illustration of the developmental stages of the mice left ventricle tissues sampled in this study and the short description of the core experiment manipulations. The statistical applications for evaluating the expression stability of reference genes were also shown. $E=$ embryonic day; $D=$ postnatal day; $M=$ postnatal month 
A
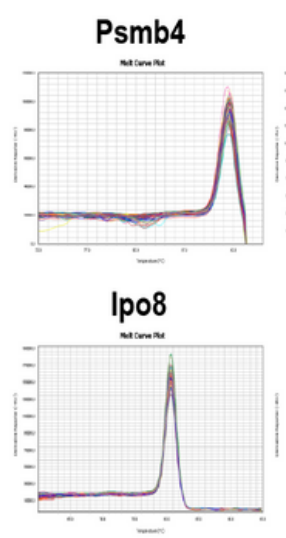

18S
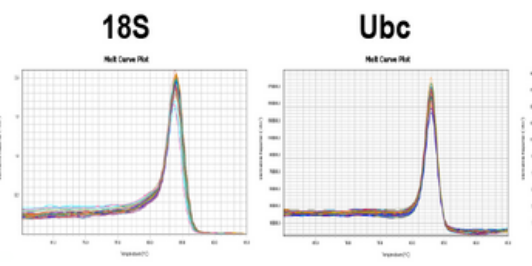

Reep5
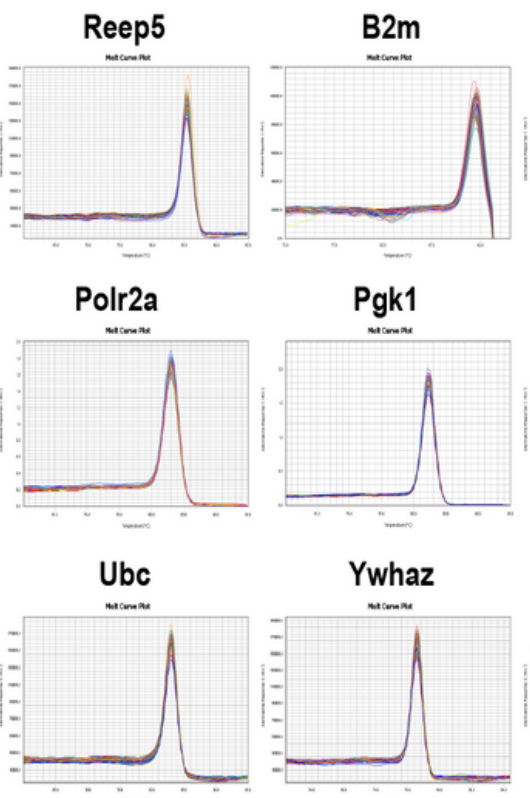

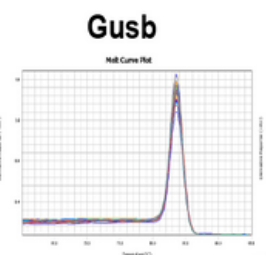

Ppia

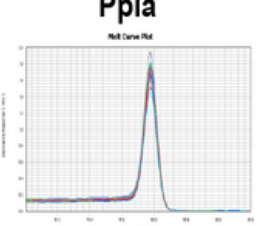

Sdha

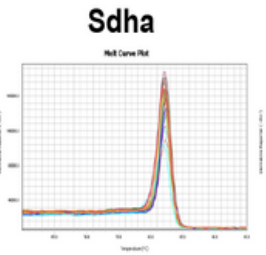

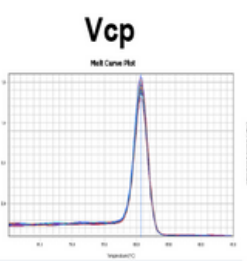
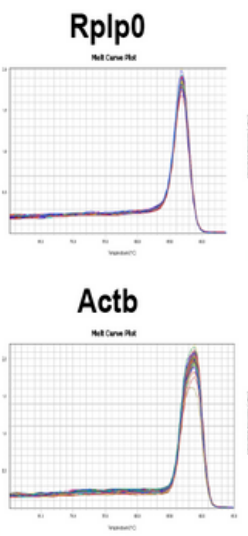
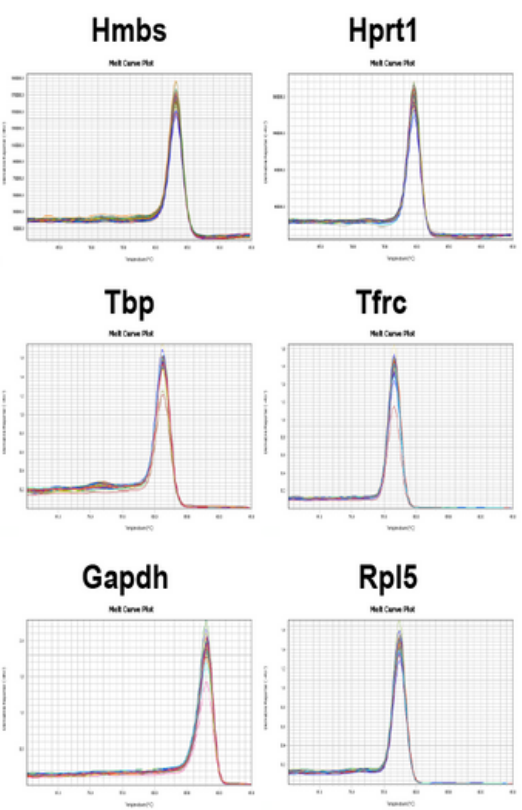

B
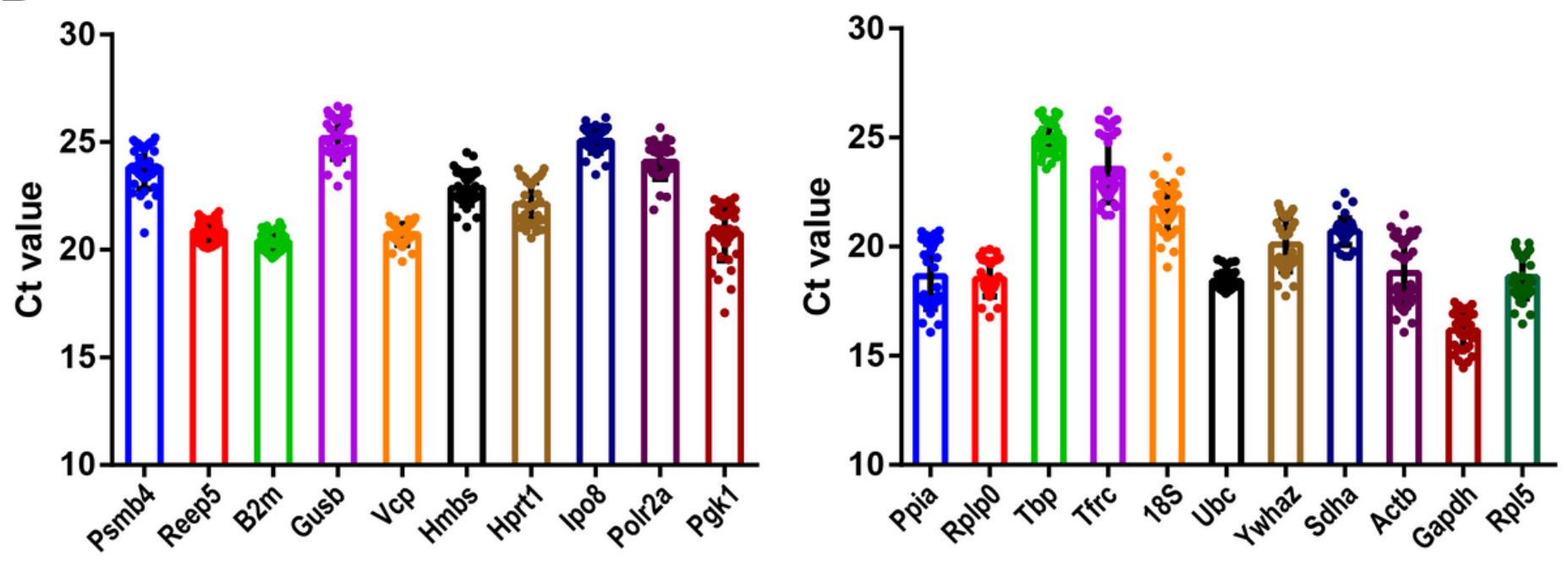

Figure 2

Overall expression characteristics of the reference genes during development of mouse heart. (A) The melting curve assays of the 21 candidate reference genes across all samples showed the single peak, indicated the RT-qPCR amplification had good specificity;

(B) The expression of cycle threshold (Ct) -values in each candidate reference gene. Bar graph representative of mean values of the Ct value. 
A

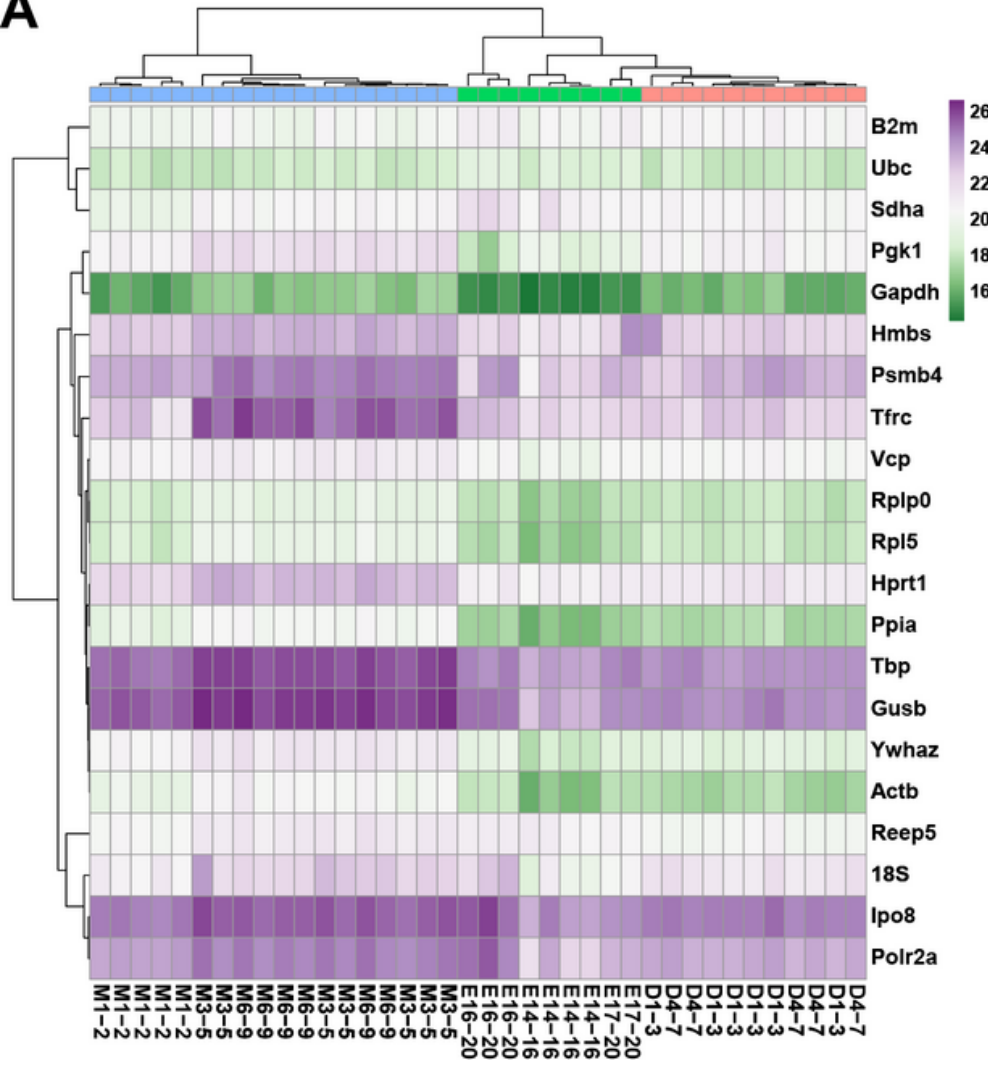

B

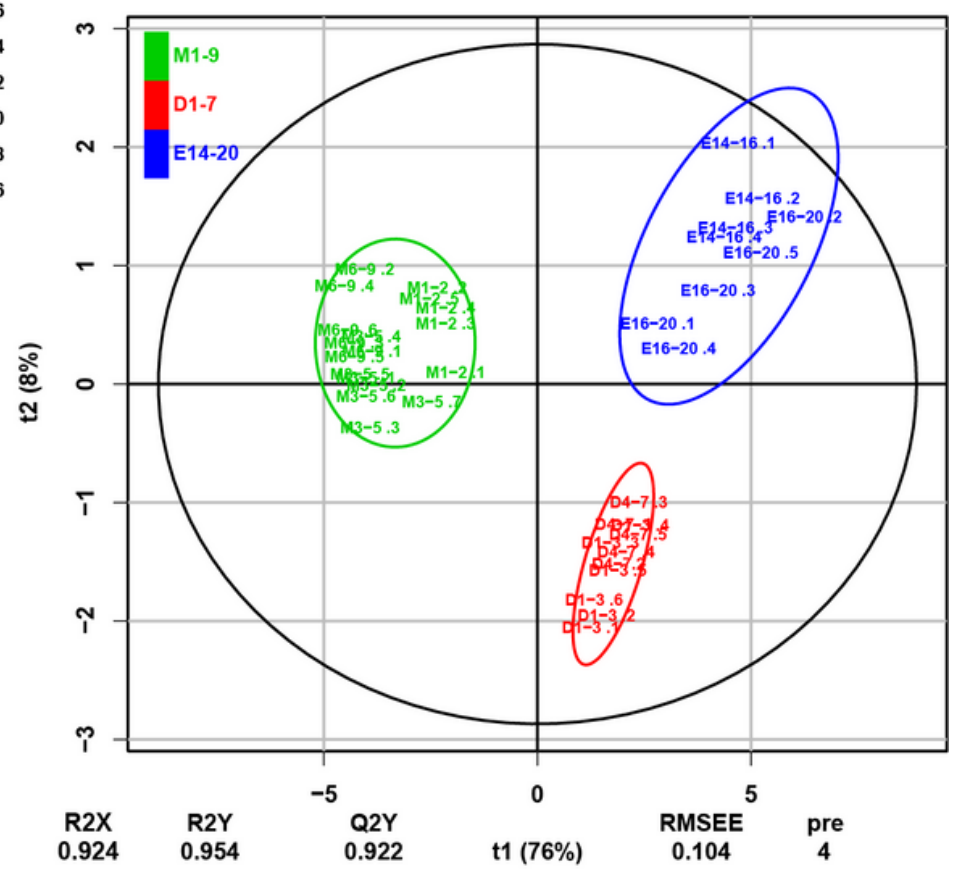

Figure 3

Clustering analysis of the candidate reference genes expression. (A) Heat map of hierarchical cluster analysis of samples performed on the profiles of 21 candidate reference genes; (B) Supervised OPLS-DA score plot. Three phases can be distinguished in the gene expression features, that are (1) embryo stage, (2) first 7 days after birth, (3) 1 to 9 months after birth. 
A

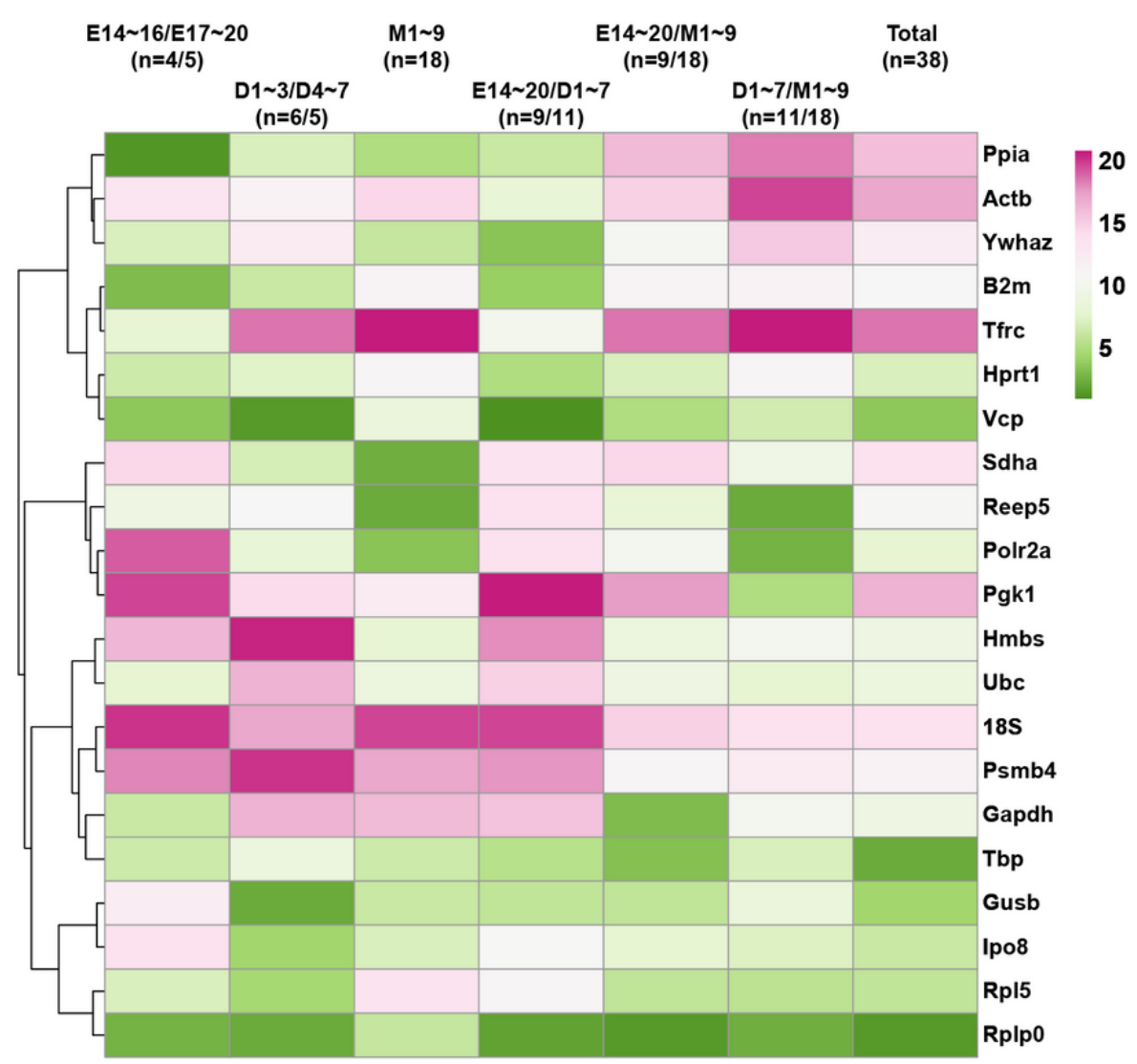

B

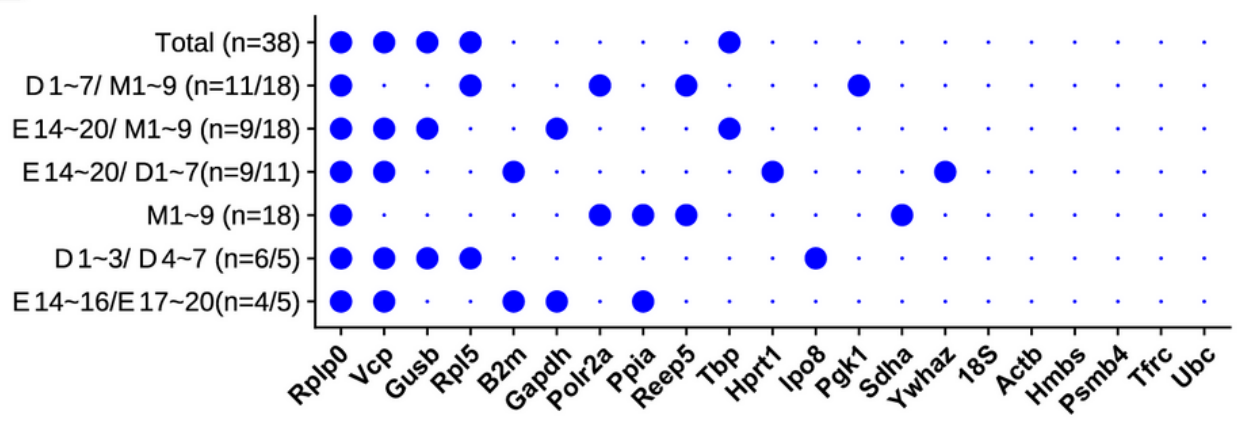

\section{Figure 4}

Stability of candidate reference genes. (A) Heatmap to illustrate the most suitable 5 reference genes with high stability in each dataset with different condition using. The color scale indicates the stability: Green color indicate higher stability, while red color represents a decreased stability. (B) Dot plot graph to show the optimal reference genes in each condition. 
A
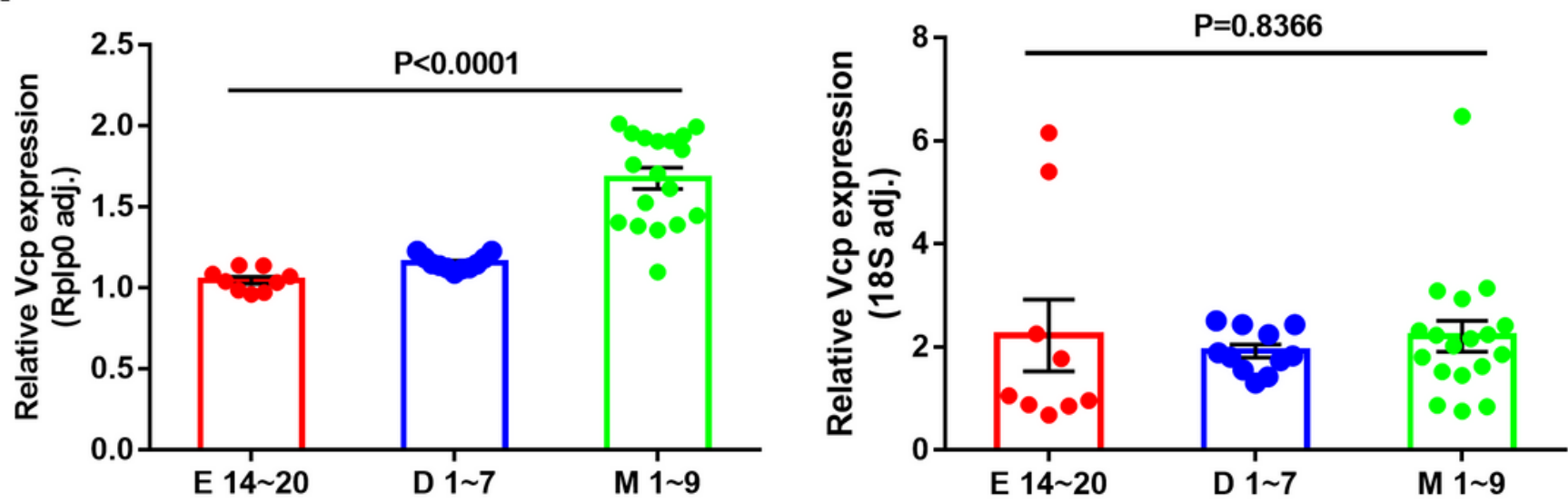

B
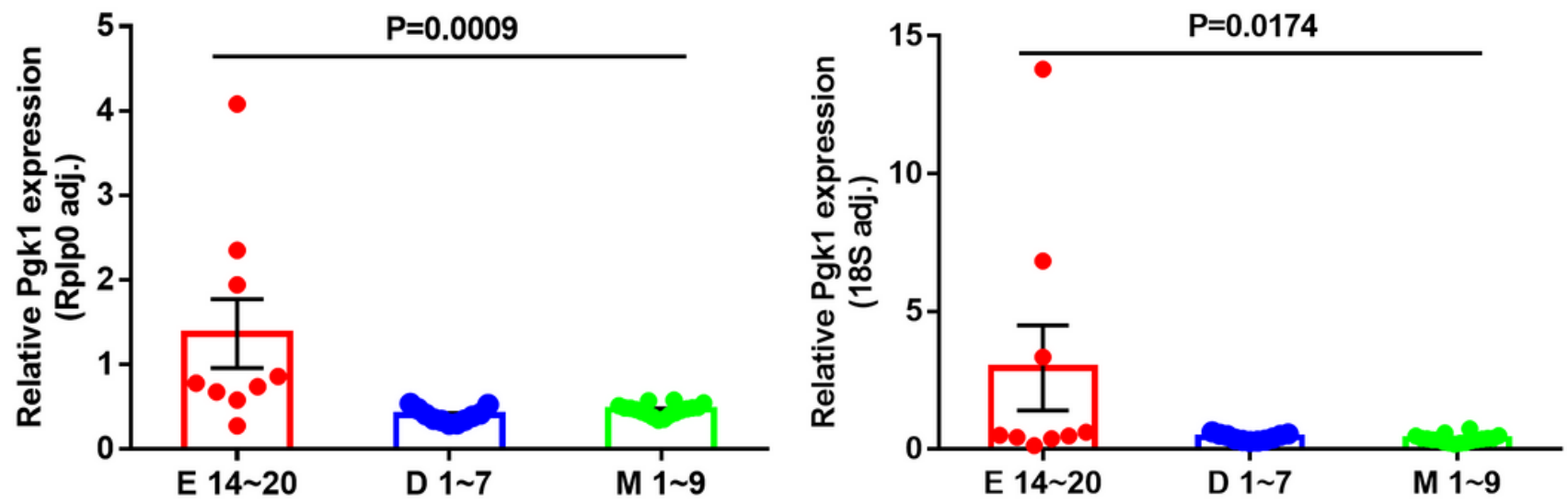

Figure 5

Relative expression of two target genes. (A) Vcp expression detection normalized by reference gene Rplp0 or 18S; (B) Pgk1 expression detection normalized by reference gene Rplp0 or 18S. to illustrate the variability in Vcp and Pgk1 expression in different heart developmental periods depending on the selection of the reference gene. 